\title{
CAPILLARY NETWORK IN SLOW AND FAST MUSCLES AND IN OXIDATIVE AND GLYCOLYTIC MUSCLE FIBRES
}

\author{
VITA ČEBAŠEK ${ }^{1}$, LUCIE KUBÍNOVÁ $^{2}$, SAMO RIBARIČ $^{3}$ AND IDA ERŽEN ${ }^{1}$ \\ ${ }^{1}$ Institute of Anatomy, Medical Faculty, University of Ljubljana, Ljubljana, Slovenia; ${ }^{2}$ Institute of Physiology, \\ Academy of Sciences of the Czech Republic, Prague, Czech Republic; ${ }^{3}$ Institute of Pathophysiology, Medical \\ Faculty, University of Ljubljana, Ljubljana, Slovenia \\ e-mail: vita.cebasek@mf.uni-lj.si; lucie.kubínova@biomed.cas.cz; samo.ribaric@mf.uni-lj.si; ida.erzen@mf.uni-lj.si \\ (Accepted February 23, 2005)
}

\begin{abstract}
The aim of this study was to compare capillary network in slow and fast muscles and also in oxidative and glycolytic muscle fibres. Soleus (SOL) and extensor digitorum longus (EDL) muscles were excised from five female rats. Capillaries and muscle fibres were demonstrated on thick tissue sections by a triple immunofluorescent method. Stacks of perfectly registered optical images were captured by a confocal microscope and further analysed. Applying stereological methods (POINTGRID, FAKIR and SLICER plugin-modules of the Ellipse programme), we estimated the mean length of capillaries, adjacent to individual muscle fibre, per unit fibre length (Lcap/Lfib), per unit surface area of the fibre (Lcap/Sfib) and per unit fibre volume (Lcap/Vfib) in the slow SOL and in predominantly fast EDL muscle, and separately in oxidative and glycolytic fibres of EDL muscle. The length of capillaries per unit fibre length was larger in SOL than in EDL muscle, however, capillary length per unit fibre volume was larger in EDL muscle. There was no difference in the length of capillaries per unit fibre surface area between the two muscles. Oxidative and glycolytic fibres differ in the length of capillaries per unit fibre surface area (Lcap/Sfib). This parameter probably reflects the oxidative capacity of muscle fibres. In conclusion, capillary supply is evidently well adapted to different muscle fibre types; consequently, an average capillary supply of a heterogeneous muscle depends on the muscle composition. The estimated mean values blur some intrinsic differences.
\end{abstract}

Keywords: capillaries, muscle fibres - oxidative and glycolytic, skeletal muscle - slow and fast, stereology.

\section{INTRODUCTION}

Capillaries supply muscles and muscle fibres with oxygen and nutrients and remove waste metabolic products (Hudlicka, 1991). Early studies of Ranvier (1874) have shown that the capillary density is higher in 'red' muscles than in 'white' muscles. Further histochemical and biochemical studies confirmed that the density of the capillary network is directly proportional to the level of the oxidative metabolism (Romanul, 1965; Gray and Renkins, 1978). As the muscle adapts to different workloads, remodelling of both, the type of metabolism and the capillary density occurs (Hudlicka, 1985; Škorjanc et al., 1998).

Skeletal muscles are usually heterogeneous, composed of fibre types that differ in their contraction velocity (Botinnelli et al., 1994) and in their resistance to fatigue ( $c f$. Pette and Staron, 2001). The higher the level of the oxidative metabolism, the more a muscle fibre or a muscle is resistant to fatigue. On the other hand, the higher the level of the glycolytic metabolism, the more a muscle fibre or a muscle is susceptible to fatigue. In mammals, at least four distinct fibre types exist: one is slow contracting, fatigue resistant, and three are fast contracting, whereby the contraction velocity gradually increases and fatigue resistance gradually decreases along the following pathway: type 2a, 2x/d, 2b (Botinnelli et al., 1994; Pette and Staron, 2001). Very few muscles are composed of only one or of predominantly one fibre type. An example of such a muscle is the slow contracting fatigue resistant rat soleus muscle (SOL). Extensor digitorum longus muscle (EDL) of the rat is a predominantly fast contracting muscle, composed of a very low percentage of slow (type) 1 oxidative fibres and a variable proportion of type $2 \mathrm{a}, 2 \mathrm{x} / \mathrm{d}$ and $2 b$ fibres.

In this work, we studied the overall capillary network in rat soleus and extensor digitorum longus muscles and the local, capillary network around oxidative fibres in both, SOL and EDL muscle, and in glycolytic fibres in the EDL muscle. We estimated 
the length of capillaries adjacent to the individual muscle fibres, related to different fibre parameters: capillary length per unit fibre length (Lcap/Lfib), per unit surface area of the fibre (Lcap/Sfib) and per unit fibre volume (Lcap/Vfib). The aim of the study was to answer the following questions:

i. Which of the parameters reflecting the capillary density are different in slow compared to fast muscles and among predominantly oxidative and predominantly glycolytic fibres?

ii. Is the capillary density of a muscle or a muscle fibre dependent on the intensity of the oxidative metabolism or is it fibre cross-sectional area that is decisive?

\section{MATERIAL AND METHODS}

Soleus and extensor digitorum longus muscles were excised from five female Wistar rats, and then deeply frozen in liquid nitrogen and cut into 10 and $180 \mu \mathrm{m}$ thick transverse sections from the middle of the muscle belly.

\section{FIBRE TYPING}

$10 \mu \mathrm{m}$ thick frozen sections of SOL and EDL muscles were stained for succinate dehydrogenase (SDH) (Reichmann and Pette, 1991), an indicator of the oxidative metabolism, to demonstrate resistance to fatigue, and $\alpha$-glycerophosphate dehydrogenase (GPDH) (Kugler, 1991), an indicator of the glycolytic metabolism, to demonstrate fast fatigability of muscle fibres. Additionally, myosin heavy chain (MyHC) isoforms, MyHC-1 (in both muscles) and MyHC-2b (in EDL muscles) to show slow contracting and the fastest contracting muscle fibres were demonstrated by the immunoperoxidase method applying antibodies BAD5 and BFF3, respectively; MyHC-2a in EDL was proved by Sc-71 (Schiaffino et al., 1989) (Figs. 1,2).
We intended to compare capillary supply of slow contracting oxidative fibres in the SOL muscle with oxidative and glycolytic fibres in the EDL muscle. As we did not succeed in superimposing another staining for fibre typing to the existing triple staining of capillaries and muscle fibres in thick muscle sections (see below) we introduced another criteria to distinguish among oxidative and glycolytic fibres in the EDL muscle. We measured fibre diameter with the Ellipse program (ViDiTo, Slovakia) in sections, stained for MyHC-2b (Fig. 2). In every muscle, fibre diameters were arranged into three groups with the Kmean method implemented in the SYSTAT statistical package (Table 1). Values are expressed in percentage of the largest fibre diameter found in the muscle sample. We checked every fibre within each of the three groups whether it was MyHC-2b positive or negative. The large fibre group contained only MyHC-2b positive fibres (see dark fibres in Fig. 2c), which means that all large fibres were glycolytic and non-oxidative (see dark fibres in Fig. 2d and pale fibres in Fig. 2e). The small fibre group contained only MyHC-2b negative fibres, and corresponded to slower contracting oxidative fibres, most of them were slow, type 1 fibres and some were fast $2 \mathrm{a}$ fibres (see dark fibres in Figs. $2 a, b, e)$. The middle fibre group was a mixed group of MyHC-2b positive and MyHC-2b negative fibres, i.e., fibres with oxidative and glycolytic metabolism (compare also Fig. 2). Fibre diameters in each group were normally distributed, no extremely large values were present. Only two groups were further analysed separately: 'large fibres' and 'small fibres' (Table 1). 'Large fibres' were those with diameters larger than the lower limit of the group and 'small fibres' were those with diameters smaller than the upper limit of the group. In this way, we successfully separated strong oxidative fibres from non-oxidative fibres, i.e., pure glycolytic fibres.

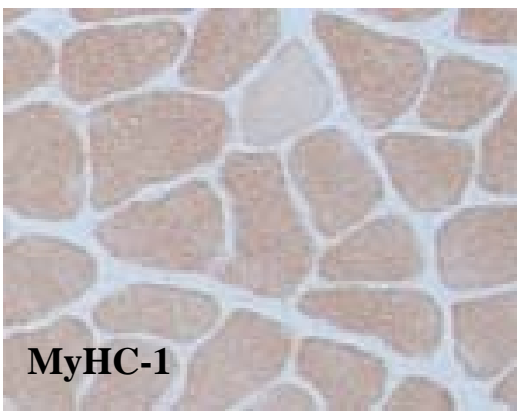

a)

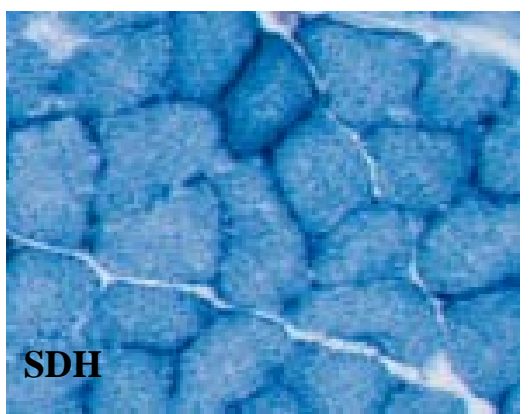

b)

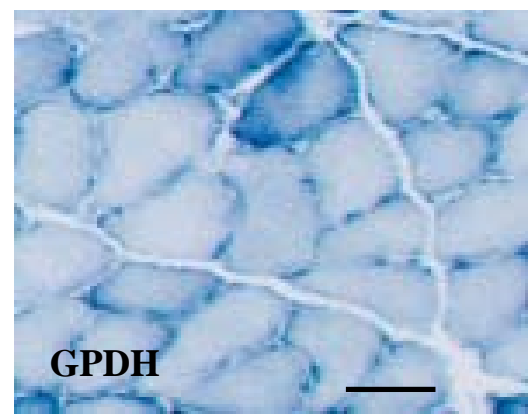

c)

Fig. 1. Fibre types in rat soleus muscle, demonstrated by the immunoperoxidase reaction against myosin heavy chain-1 (MyHC-1) (a), succinate dehydrogenase (b) and activity of $\alpha$-glycerophosphate dehydrogenase (c). Dark fibres in (a) are type 1, dark fibres in (b) are oxidative and dark fibres in (c) are glycolytic fibres. Bar: $50 \mu m$. 


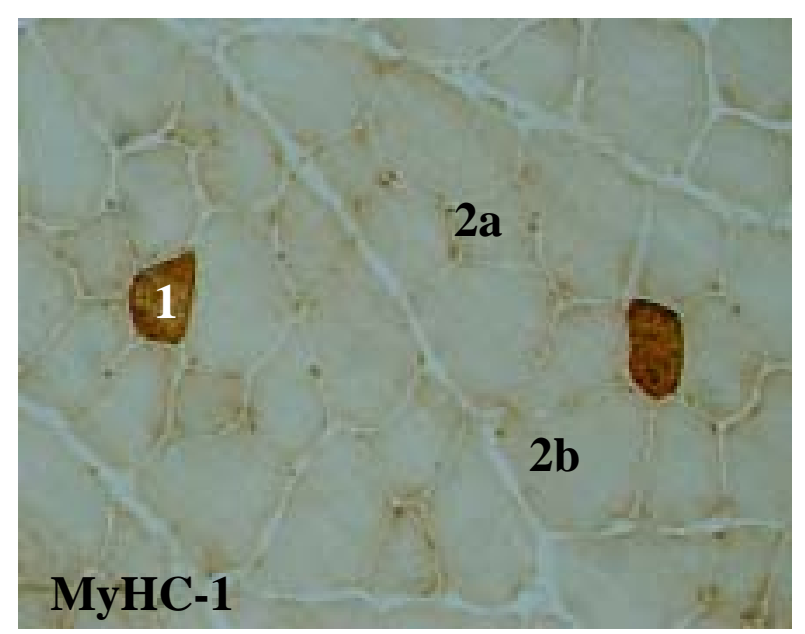

a)

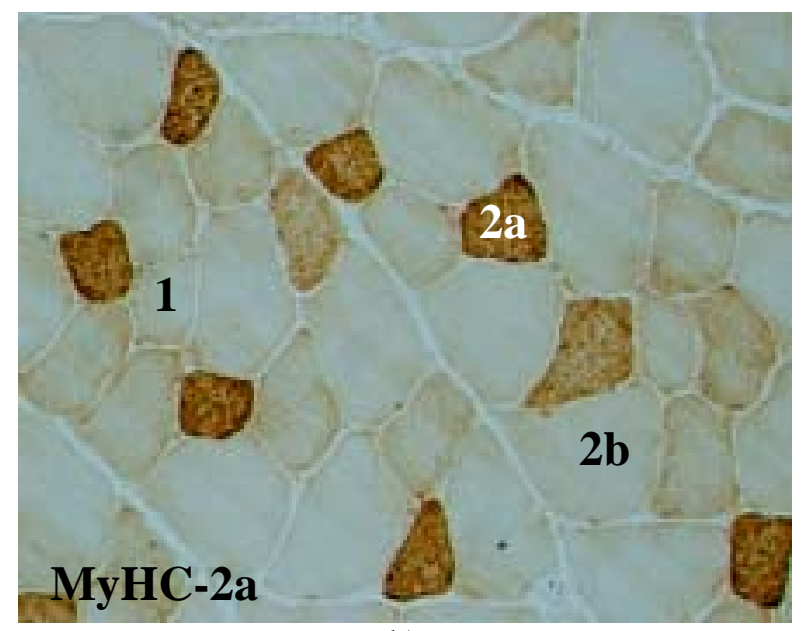

b)

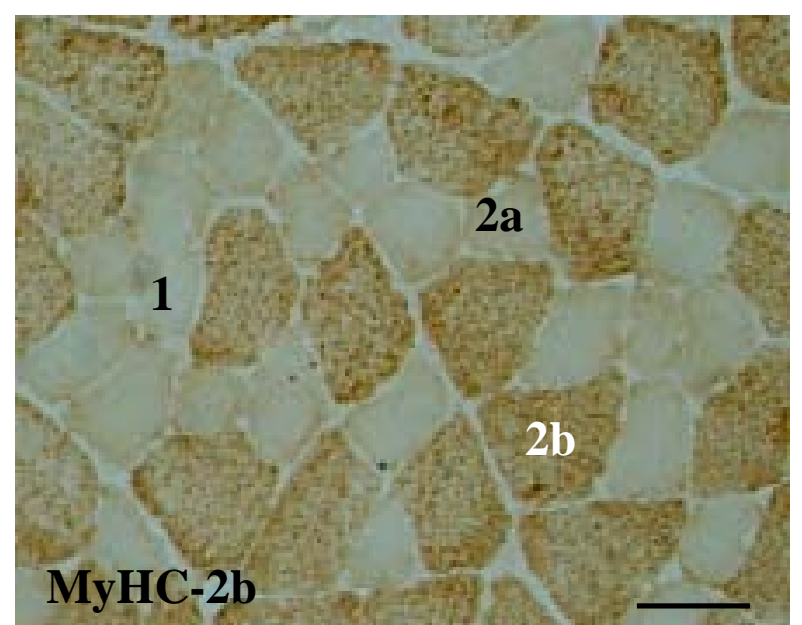

c)

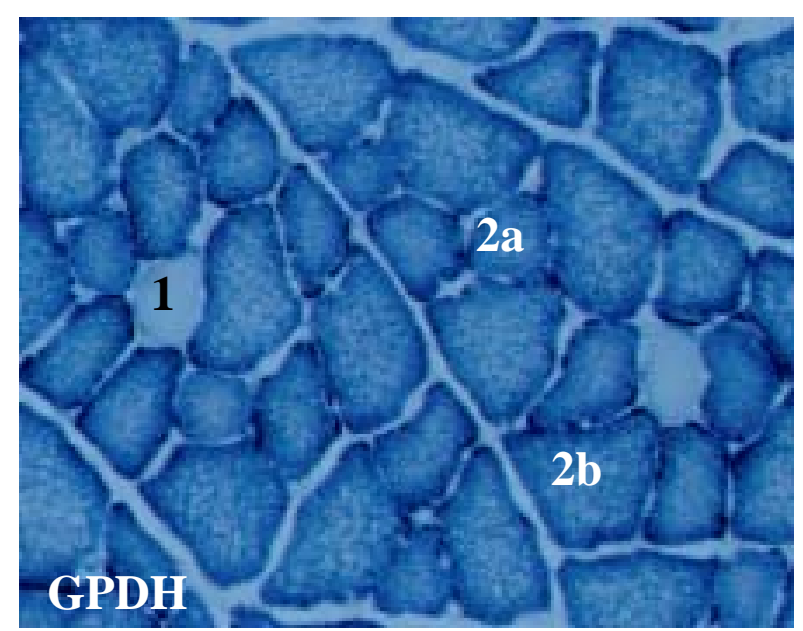

d)

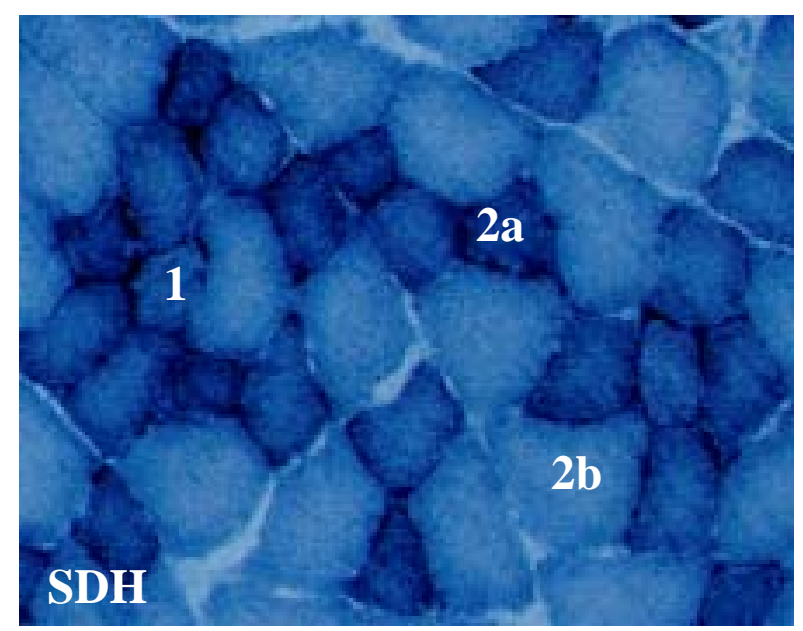

e)

Fig. 2. Fibre types in the extensor digitorum longus muscle, demonstrated by the immunoperoxidase reaction against different myosin heavy chain (MyHC) isoforms (a-c), activity of $\alpha$-glycerophosphate dehydrogenase (d) and succinate dehydrogenase (e). Fibres marked 1 contain MyHC-1, 2a contain MyHC-2a, $2 b$ contain MyHC2b. Dark fibres in (d) are glycolytic fibres, dark fibres in (e) are oxidative fibres. Bar: $50 \mu \mathrm{m}$. 
Table 1. Distribution of fibres in five EDL muscles into three groups according to fibre diameter. For each muscle and for each group, average, lower and upper limits are expressed in percentage of the largest diameter found in the given muscle [\%].

\begin{tabular}{c|ccc}
\hline & \multicolumn{3}{|c}{$\begin{array}{c}\text { Mean of fibre diameter proportion [\%] in fibre groups in EDL muscle } \\
\text { (minimum }\end{array}$} \\
\hline EDL muscle No. & small fibres & middle fibres & large fibres \\
\hline \multirow{2}{*}{1} & 49 & 65 & 84 \\
& $(31-57)$ & $(57-74)$ & $(74-100)$ \\
2 & 43 & 61 & 78 \\
& $(27-52)$ & $(52-69)$ & $(70-100)$ \\
3 & 41 & 60 & 74 \\
& $(25-50)$ & $(50-67)$ & $(67-100)$ \\
4 & 45 & 67 & 86 \\
& $(29-55)$ & $(56-76)$ & $(76-100)$ \\
5 & 46 & 72 & 87 \\
& $(31-59)$ & $(59-79)$ & $(79-100)$ \\
\hline
\end{tabular}

\section{STAINING OF CAPILLARIES AND MUSCLE FIBRES}

Capillaries and muscle fibres of SOL and EDL muscles were demonstrated in thick frozen sections by the triple immunofluorescent staining (Čebašek et al., 2004): basal lamina of muscle fibres was demonstrated with antibodies against laminin (in red) whilst capillaries were double stained with antibodies against CD31 and with lectin Griffonia (Bandeira) simplicifolia I (both in green).

\section{SAMPLING}

From each muscle cross-section, five systematically sampled stacks of 41 perfectly registered optical images with $x-y$ dimensions of $375 \mu \mathrm{m} \times 375 \mu \mathrm{m}$ $(512 \times 512$ pixels $), 1 \mu \mathrm{m}$ apart, were captured by Leica SP2 AOBS confocal microscope applying oil immersion $40 \times$ objective (NA 1.25).

Fibres were sampled by an unbiased counting frame (Gundersen, 1977), on average about 23 in the EDL and about 11 in SOL. Further, capillary length was measured adjacent to each sampled fibre.

\section{STEREOLOGICAL ESTIMATION OF CAPILLARY DENSITY}

Volume and surface area of the individual sampled fibres within stacks of optical sections, as well as the length of capillaries supplying individual fibres were estimated by stereological methods applying the 'POINTGRID', 'FAKIR' and 'SLICER' plug-in modules of the Ellipse programme system (Tomori, 2002). Details of the methods are explained elsewhere (Kubínová and Janáček, 1998; Kubínová et al., 2001).
Briefly, the length of capillaries (Lcap) adjacent to individual fibres was estimated by counting transections of capillaries with a spatial grid consisting of three systems of parallel equidistant virtual test planes, perpendicular to each other, applying the SLICER method (Larsen et al., 1998). The surface area of each muscle fibre in the given stack (Sfib) was estimated by counting intersections of the fibre surface with a cubic spatial grid consisting of three mutually perpendicular FAKIR probes, i.e., parallel test lines resembling nails of a fakir bed piercing the surface (Kubínová and Janáček, 1998). The volume of fibres was estimated using the spatial grid of points (Cruz-Orive, 1997). In both fakir and slicer methods, isotropic uniform random (IUR) orientation of the virtual spatial grid consisting of fakir or slicer probes, respectively, was guaranteed by a random generation of the grid origin and a random rotation of the grid (for details see Kubínová et al., 2002) using the special FAKIR and SLICER programme modules mentioned above. We estimated the following parameters: length of capillaries adjacent to individual fibres per unit fibre length (Lcap/Lfib), per unit fibre surface area (Lcap/Sfib), and per unit fibre volume (Lcap/Vfib).

\section{STATISTICS}

We calculated the mean fibre length, volume and surface area within a $40 \mu \mathrm{m}$ thick section of an average fibre in the SOL and EDL as well as Lcap/Lfib, Lcap/Sfib and Lcap/Vfib and standard deviation of mean. Additionally, the same parameters were estimated for the following muscle fibre types: slow oxidative in the SOL, oxidative (small fibres) and fast glycolytic (large fibres) in the EDL muscle. 
Data for each muscle and muscle fibre type were tested with the Student $\mathrm{t}$-test with the Bonferonni correction, applying the SYSTAT statistical package.

In the soleus muscle, we analysed all fibres that were sampled by the unbiased counting frame, assuming that most of the fibres were slow contracting oxidative.

In the EDL muscle, however, strong oxidative and glycolytic, i.e., non-oxidative fibres were selected by fibre volume measured within the $40 \mu \mathrm{m}$ thick stacks of optical images. Based on the upper limits of fibre profiles, selected by their diameter, in the 'small fibre group' and on lower limits of fibre profiles 'in the large fibre group' determined on thin transverse sections (see Table 1), we separated 'large' fibres from 'small' fibres by their volume in a $40 \mu \mathrm{m}$ thick stack of optical images.

\section{RESULTS}

\section{FIBRE TYPES IN THE RAT SOLEUS AND EXTENSOR DIGITORUM LONGUS MUSCLE}

Rat soleus muscle is a homogeneous slow twitch muscle composed of over 90 percent of slow twitch type 1 fibres (BAD5 positive), that exhibit high oxidative (SDH) and low glycolytic (GPDH) metabolism (Fig. 1). However, EDL muscle is a predominantly fast twitch muscle, composed of at least three fast twitch fibre types, $2 \mathrm{a}, 2 \mathrm{x} / \mathrm{d}$ (not shown) and $2 \mathrm{~b}$ and of a minority of slow twitch fibres. The fibre types differ in their predominant metabolism and fibre diameter (Fig. 2). Type 1 fibres are the smallest (BAD5 positive) with high activity of SDH, and very low activity of GPDH, type $2 b$ fibres are the largest (BFF3 positive) with high GPDH and very low SDH activity.

\section{IMMUNOFLUORESCENT DEMONSTRATION OF CAPILLARIES AND MUSCLE FIBRES}

Green and yellow stained capillaries with red stained basal lamina of muscle fibres in the rat SOL and EDL muscles are presented in Fig. 3.

\section{CAPILLARY DENSITY IN SLOW AND FAST MUSCLES}

Length of capillaries adjacent to individual fibres estimated per unit fibre length (Lcap/Lfib), per unit surface area of the fibre (Lcap/Sfib) and per unit fibre volume (Lcap/Vfib) in the SOL and EDL muscles are presented in Table 2. Lcap/Lfib was larger in the SOL than in the EDL muscle, Lcap/Vfib was larger in the EDL than in the SOL, and Lcap/Sfib was similar in both muscles. Surface area of the fibre (S), and fibre volume (V) within $40 \mu \mathrm{m}$ thick sections are larger in the SOL than in the EDL muscle (Table 2).

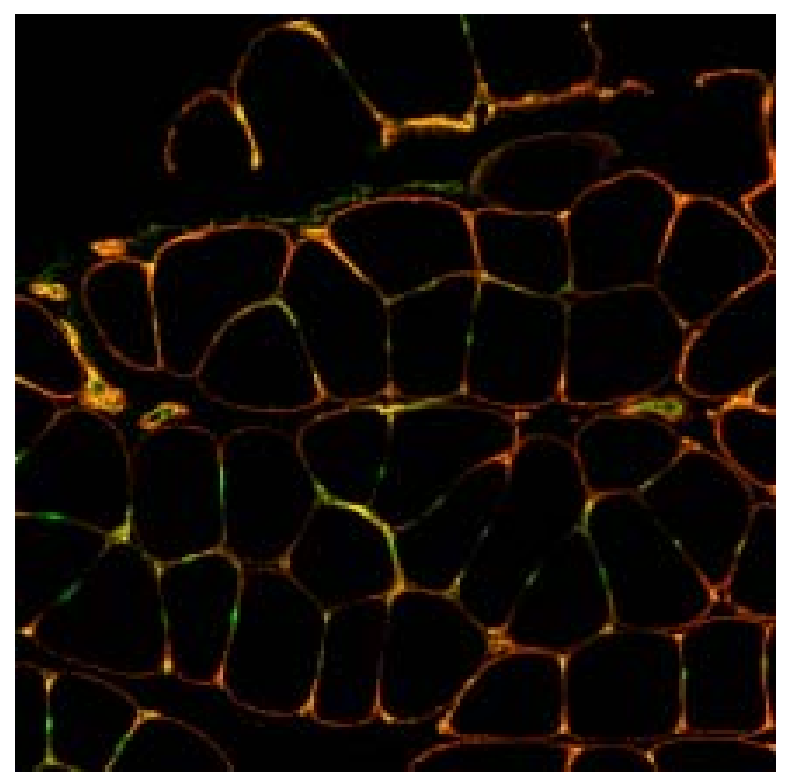

a)

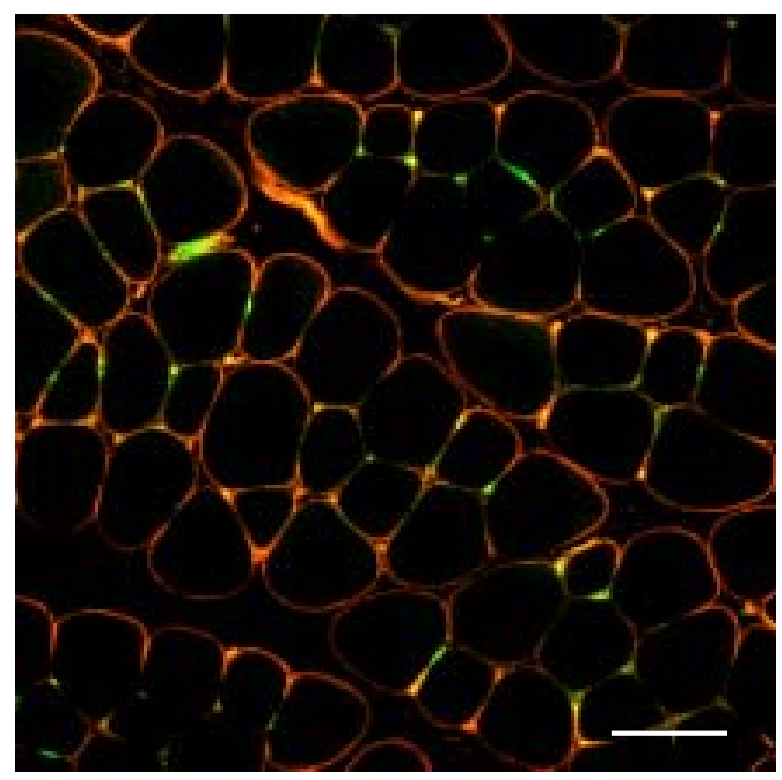

b)

Fig. 3. Capillaries in rat soleus (a) and extensor digitorum longus (b) muscle. Bar:50 $\mu m$. 
Table 2. Stereological characteristics of capillaries and muscle fibres within $40 \mu \mathrm{m}$ thick muscle cross sections (average $\pm S D$ ). Mean surface area (Sfib) and volume (Vfib) of the fibres within stacks of optical sections, length of capillaries adjacent to individual fibres per unit fibre length (Lcap/Lfib), per unit surface area of the fibre (Lcap/Sfib) and per unit fibre volume (Lcap/Vfib).

\begin{tabular}{|c|c|c|}
\hline & Muscles & Fibres in EDL \\
\hline & EDL & Large fibres \\
\hline$\overline{\text { Sfib }\left[\mu \mathrm{m}^{2}\right]}$ & $8643 \pm 1244^{*}$ & $7745 \pm 1357$ \\
\hline & $\mathrm{p}<0.05$ & $\mathrm{p}<0.05$ \\
\hline Vfib $\left[\mu \mathrm{m}^{3}\right]$ & $\begin{array}{c}141667 \pm 35498^{*} \quad 83791 \pm 18922 \\
p<0.05\end{array}$ & $\begin{array}{c}38852 \pm 12847^{*} \quad 126580 \pm 46212 \\
\mathrm{p}<0.05\end{array}$ \\
\hline Lcap/Lfib & $\begin{array}{c}6.91 \pm 1.56^{*} \\
p<0.05\end{array}$ & $\begin{array}{lll}4.31 \pm 0.64^{*} & & 4.84 \pm 1.64 \\
& \text { n.s. } & \\
\end{array}$ \\
\hline Lcap/Sfib $\left[\mathrm{mm}^{-1}\right]$ & $\begin{array}{cc}31.37 \pm 3.43 * \pm & \\
\text { n.s. } & \\
& \end{array}$ & $\begin{array}{c}39.03 \pm 0.99 * \\
\mathrm{p}<0.05\end{array}$ \\
\hline Lcap/Vfib $\left[\mathrm{mm}^{-2}\right]$ & $\begin{array}{c}2029 \pm 170^{*} \\
p<0.05\end{array}$ & $\begin{array}{c}4625 \pm 865^{*} \\
p<0.05\end{array}$ \\
\hline
\end{tabular}

$* \mathrm{p}<0.05$ (fibres in the soleus muscle were compared to small fibres in the EDL) $\$ \mathrm{p}<0.05$ (fibres in the soleus were compared with large fibres in the EDL)

\section{CAPILLARY DENSITY IN OXIDATIVE FIBRES IN SLOW AND FAST MUSCLES AND IN GLYCOLYTIC FIBRES OF THE FAST MUSCLE}

Capillary density in slow twitch oxidative fibres in the SOL muscle, i.e., in all SOL fibres, in oxidative (small fibres) and fast twitch glycolytic (large fibres) fibres in the EDL and fibre volume and surface area within $40 \mu \mathrm{m}$ thick frozen sections are presented in Table 1.

Lcap/Lfib is larger in the slow oxidative SOL muscle fibres than in the EDL muscle in either oxidative (small fibres) or in glycolytic fibres. In oxidative fibres of both SOL and EDL muscle, Lcap/Sfib is larger than in glycolytic fibres (large) in the EDL muscle. Small oxidative fibres in the EDL exhibit even larger values than oxidative fibres in the SOL. The fibre surface area, however, is much larger in the oxidative fibres of the SOL than in the oxidative fibres of EDL muscle and it is nearly equal to the surface area of fast glycolytic fibres of the EDL muscle. Lcap/Vfib is the largest in the oxidative fibres of the EDL muscle and the smallest in the glycolytic fibres of the EDL muscle. Slow, oxidative SOL muscle fibres and fast, glycolytic (large) EDL fibres have nearly equal fibre volume. Both have a much larger volume than oxidative (small) fibres in the EDL muscle.

\section{DISCUSSION}

In this study, the density of the capillary network in skeletal muscles was studied from stacks of perfectly registered optical images captured by a confocal microscope. Stereological methods and the 3D approach used in our study enable to measure correctly the length of capillary network around each muscle fibre, including anastomoses, therefore our method is more appropriate than the most often used 2D approach on transverse muscle sections, i.e., sections with fixed orientation. However, since most capillaries are running along muscle fibres, it is reasonable to compare our data on the capillary supply in different muscles with those obtained from 2D, as the obtained values can follow a similar trend.

We introduced several stereological parameters and compared them with already published parameters, whereby to our knowledge, the capillary supply in rat SOL and EDL muscles was estimated by a stereological approach only in few published papers (MathieuCostello et al., 1996; Xu et al. 1998).

The parameter Lcap/Lfib points to a better capillary supply in the SOL than in the EDL and shows no difference between predominantly oxidative and predominantly glycolytic fibres in the EDL. We anticipate that in the EDL muscle higher proportion of capillaries runs parallel to the muscle fibre longitudinal 
axes than in SOL. This parameter discriminates the best between the slow soleus and fast EDL muscle and seems to be genetically predetermined. Larger Lcap/ Lfib in SOL than in EDL is in accordance with the number of capillaries per fibre number $(\mathrm{C} / \mathrm{F})$ and number of capillaries around fibres (CAF) measured in 2D (Hansen Smith et al.,1992; Deveci et al., 2001).

Larger Lcap/Vfib in EDL is connected with the smaller mean fibre diameter in the EDL muscle. The largest fibres in the EDL, that comprise about $50 \%$ of all fibres, reach about the same diameter as the average fibre in the SOL. The parameter Lcap/Vfib shows higher values for fibres in the EDL than in SOL. It depends on the share of different fibre types in the EDL that varies from muscle to muscle and it varies also along the length of the EDL muscle (Williams and Segal, 1992). The larger Lcap/Vfib in small (predominantly oxidative) than in large (predominantly glycolytic) fibres in the EDL is due to the higher oxidative metabolism and smaller fibre diameter. Larger Lcap/Vfib in the EDL than in SOL is in accordance with the number of capillaries per unit cross-sectional area (Deveci et al., 2001) measured in 2D.

Estimated values for Lcap/Vfib in the soleus muscle are nearly equal to the capillary length per unit volume, estimated using the Dimroth-Watson axial distribution and reported by $\mathrm{Xu}$ et al. (1998). However, in EDL muscle the reported values (Mathieu-Costello et al., 1996) are lower than in this study. This discrepancy is probably based on the different ratio of fibre types within EDL muscles studied as well as on the sampling procedure, as the ratio among fibre types changes along the muscle length (Wang and Kernell 2000).

The parameter Lcap/Sfib appears to correlate well with the oxidative capacity of fibres, a feature that depends on the subsarcolemmal deposits of mitochondria. This parameter has nearly equal values in SOL and EDL which corresponds with the activity of citrate synthase, an indicator of the oxidative metabolism, reported to be nearly equal in homogenates of both muscles (Bass et al., 1983). Higher Lcap/Sfib values in oxidative fibres compared to glycolytic fibres and even higher values in small (oxidative) fibres in EDL than in oxidative fibres in SOL are also in accordance with the activity of enzymes of the oxidative metabolism measured histophotometrically (Punkt et al., 1996; Škorjanc et al., 1998).
Capillary supply is evidently well adapted to different muscle fibre types; consequently, an average capillary supply of a heterogeneous muscle depends on the muscle composition. The estimated mean values blur some intrinsic differences. However, average values for a muscle can successfully be correlated with biochemical data obtained from muscle homogenates.

In conclusion, capillary density appears to reflect the oxidative capacity of muscles and their constituting muscle fibres. In this study we have proved that the volume and surface area of oxidative fibres in the soleus muscles is nearly equal to the volume and surface area of glycolytic fibres in the EDL, however, capillary density, expressed by all estimated parameters is much larger in the oxidative SOL fibres than in the glycolytic EDL fibres. This supports the hypothesis that it is not only the fibre diameter that dictates capillary density but also the fibre's oxidative capacity. On the other hand, in the EDL, fibre diameter is related to the activity of oxidative enzymes, the smallest fibres express the highest activity and the largest the lowest activity. However, whether it is the fibre diameter that conditions the oxidative activity or vice versa, remains an open question. Relation between fibre diameter and the activity of the oxidative metabolism has been proved also in other muscles (Nemeth and Pette, 1981; Armstrong and Phelps, 1984; Egginton, 1990; Rivero et al., 1998). Based on our results, among parameters introduced in this study it seems that parameters Lcap/Lfib and Lcap/Vfib, are more dependent on fibre diameter than Lcap/Sfib in all types of fibres (compare also Kubínová et al., 2001). Lcap/Sfib reflecting the fibre oxidative capacity better than Lcap/Lfib and Lcap/Vfib, thus appears to be the most suitable parameter for characterization of fibres with different metabolism. On the other hand, Lcap/Lfib seems to be characteristic for the muscle type as it significantly differs between SOL and EDL muscle as well as among SOL and small and large fibres in the EDL. However, to prove this hypothesis different muscles with different function need to be studied.

\section{ACKNOWLEDGEMENT}

This study was supported by the Slovenian Research Agency and the Ministry of Education, Youth and Sport of the Czech Republic (KONTAKT grant No. 19/2005). 


\section{REFERENCES}

Armstrong RB, Phelps RO (1984). Muscle fibre type composition of the rat hindlimb. Am J Anat 171: 259-72.

Bass A, Teisinger J, Hnick P, Mackova E, Vejsada R, Eržen I (1983). Changes of lysosomal and energy-supply enzymes in hypertrophying muscles after denervation. Physiol Bohemoslov 32:506.

Bottinelli R, Betto R, Schiaffino S Reggiani C (1994). Maximum shortening velocity and coexistance of myosin heavy chain isoforms in single skinned fast fibres of rat skeletal muscle. J Muscle Res Cell Motil 15:413-9.

Čebašek V, Kubínová L, Ribarič S, Eržen I ( 2004). A novel staining method for quantification and $3 \mathrm{D}$ visualisation of capillaries and muscle fibres. Eur J Histochem 48: 151-8.

Cruz-Orive LM (1997). Stereology of single objects. J Microsc 186:93-107.

Deveci D, Marshall JM, Egginton S (2001). Relationship between capillary angiogenesis, fiber type, and fiber size in chronic systemic hypoxia. Am J Physiol Heart Circ Physiol 281:H241-52.

Egginton S (1990). Numerical and areal density estimates of fibre type composition in a skeletal muscle (rat extensor digitorum longus). J Anat 168:73-80.

Gray SD, Renkin EM (1978). Microvascular supply in relation to fiber metabolic type in mixed skeletal muscles of rabbits. Microvasc Res 16:406-25.

Hansen-Smith F, Banker K, Morris L (1992). Alternative histochemical markers for skeletal muscle capillaries: a statistical comparation among three muscles. Microvasc Res 44(1):112-6.

Hudlicka O (1985). Development and adaptability of microvasculature in skeletal muscle. J Exp Biol 115: 215-28.

Hudlicka O (1991). What makes blood vessels grow? J Physiol 444:1-24.

Kubínová L, Janáček J, Krekule I (2002). Stereological methods for estimating geometrical parameters of microscopical structure studied by three-dimensional microscopical techniques. In: Diaspro A, ed. Confocal and Two-Photon Microscopy. New York: Wiley-Liss, pp. 299-332.

Kubínová L, Janáček J, Ribarič S, Čebašek V, Eržen I (2001). Three-dimensional study of the capillary supply of skeletal muscle fibres using confocal microscopy. J Muscle Res Cell Motil 22:217-27.

Kubínová L, Janáček J (1998). Estimating surface area by isotropic fakir method from thick slices cut in arbitrary direction. J Microsc 191:201-11.

Kugler P (1991). Microphotometric determination of enzymes in brain sections. V. Glycerophosphate dehydrogenases. Histochemistry 95:579-83.
Larsen JO, Gundersen HJG, Nielsen J (1998). Global spatial sampling with isotropic virtual planes: estimators of lenght density and total length in thick, arbitrarily orientated sections. J Microsc 191:238-48.

Mathieu-Costello O, Agey PJ, Wu L, Hang J, Adair TH (1996). Capillary-to-fiber surface ratio in rat fast-twitch hindlimb muscles after chronic electrical stimulation. J Appl Physiol 80(3):904-9.

Nemeth P, Pette D (1981). Succinate dehydrogenase activity in fibres classified by myosin ATPase in three hind limb muscles of rat. J Physiol 320:73-80.

Pette D, Staron RS (2001). Transitions of muscle fiber phenotypic profiles. Histochem Cell Biol 115:359-72.

Punkt K, Unger A, Welt K, Hilbig H, Schaffranietz L (1996). Hypoxia-dependent changes of enzyme activities in different fibre types of rat soleus and extensor digitorum longus muscles. A cytophotometrical study. Acta Histochem (Jena) 98:255-69.

Ranvier L (1874). Note sur les vaisseax sanguins et la circulation dans les muscles rouges. Comptes Rendues hebdomadares des Seances et Memoires de la Societe de Biologie 26:28-31.

Reichmann H, Pette D (1982). A comparative Microphotometric study of succinate dehydrogenase activity levels in Type I, IIA and IIB fibres of mammalian and human muscles. Histochemistry 74:27-41.

Rivero JL, Talmadge RJ, Edgerton VR (1998). Fibre size and metabolic properties of myosin heavy chain-based fibre types in rat skeletal muscle. J Muscle Res Cell Motil 19:733-42.

Romanul FCA (1965). Capillary supply and metabolism of muscle fibres. Arch Neurol 12:497-509.

Schiaffino S, Gorza L, Sartore S, Saggin L, Ausoni S, Vianello M, Gundersen K, Lomo T (1989). Three myosin heavy chain isoforms in type 2 skeletal muscle fibres. J Muscle Res Cell Motil 10(3):197-205.

Škorjanc D, Jaschinski F, Heine G, Pette D (1998). Sequential increases in capillarization and mitochondrial enzymes in low-frequency-stimulated rabbit muscle. Am J Physiol 274(3):C810-8.

Tomori Z (2002). Ellipse version 2.01. ViDiTo, Košice, Slovakia

Wang LC, Kernell D (2000). Proximo-distal organization and fibre type regionalization in rat hindlimb muscles. J Muscle Res Cell Motill 21:587-98.

Williams DA, Segal SS (1992). Microvascular architecture in rat soleus and extensor digitorum longus muscles. Microvasc Res 43:192-204.

Xu L, Poole DC, Musch T (1998). Effect of heart failure on muscle capillary geometry: implications for $\mathrm{O}_{2}$ exchange. Med Sci Sports Exerc 30(8):1230-7. 